\title{
Subject Index to Volume 34
}

Abetalipoproteinemia, molecular genetics of, 403

Academic pediatrician, development of, 397 Acetate

colonic, in circulating acetate pool of infant pig, 318

influence on sodium transport, 666

Acetylhydrolase, platelet-activating factor, role in prevention of necrotizing enterocolitis, 237

Acidemias

D- and L-2-hydroxyglutaric, detection and prenatal diagnosis of, 277

hypoxic, effects on left ventricular endsystolic elastance in fetal sheep, 38

Acquired immunodeficiency syndrome, immune complexes in, 781

Acyl-CoA dehydrogenase, very-long-chain, deficiency of, identification of, 111

Adenosine triphosphatase, sodium, postassium, in developing tubule, effect of dopamine on, 308

Adenosine triphosphate, production in newborn hearts, source of, 735

Adenylate cyclase, in developing tubule, effect of dopamine on, 308

Adipose tissue, subcutaneous, estimation of, in infants, 448

Adolescents, iron deficiency in, 680

Adrenal hyperplasia, congenital, salt-wasting, $3 \beta$-hydroxysteroid dehydrogenase gene mutation in, 698

$\alpha_{1} B$-Adrenoceptor, developmental regulation of, in kidney, 124

Adrenocorticotropin secretion, inhibitory effect of glucocorticoids on, 646

Adrenoreceptor, intrapulmonary maturation of, 591

Albumin, regulation of lactate metabolism by, 709

Alveoli, formation in rat lung, 334

American Pediatric Society, Presidential Address, 393

Amino acids, excitatory and inhibitory, effect of experimental $E$. coli meningitis on, 187

3-Aminobenzamide, effects on nucleotide depletion, 572

Androgen receptor, influence on regulation of hepatitis B virus gene expression, 300

Anemia of prematurity, erythropoietin treatment in, 675

Antibodies

anti-HSP60, in juvenile chronic arthritis, 424

anti-TNF, and nitric oxide suppression, 345

to glutamic acid decarboxylase, in insulindependent diabetes mellitus, 785

treatment of respiratory syncytial virus infection with, 167

$\alpha_{1}$-Antichymotrypsin, synthesis by trophoblast, 312

Antioxidant enzymes

effects on nucleotide depletion, 572

fetal lung, effect of EGF on, during hyperoxia, 577

perinatal lung, differential gene expression in, in rat, 27
$\alpha_{1}$-Antitrypsin, synthesis by trophoblast, 312

Apnea

during recovery from hyperthermia, 518

relationship between bradycardia and hypoxemia and, in preterm infants, 144

Apolipoprotein(a) phenotypes, in chronic renal disease, 772

Apolipoprotein B, familial defective, molecular genetics of, 403

Apolipoprotein CII deficiency, molecular genetics of, 403

Appropriate for gestational age babies, placental transport in, 661

Arterial blood pressure, mean, influence on cerebral blood flow velocity in preterm infants, 173

Arterial partial pressure of oxygen, changes in, effect of prolonged hypoxemia on ventilatory response to, in lambs, 821

Arthritis

juvenile chronic, anti-HSP60 antibodies in, 424

juvenile rheumatoid

immune complexes in, 781

$\alpha_{2}$-MG complexes in, 204

Asphyxia

fetal, flunarizine after, neuroprotective properties of, 379

in newborns, resuscitation with $21 \%$ or $100 \% \mathrm{O}_{2}, 809$

superoxide in, 366

Assisted mechanical ventilation, with elastic unloading, 600

Autoantigens, fibroblast-derived CD, 420

Autonomic state, fetal, spectral analysis, 134

Bactericidal activity, lung, and pulmonary hypertension, 32

Barness, Lewis A., Howland Award presentation to, 551

Benzodiazepine receptors, peripheral-type, binding sites for, 777

Bilirubin toxicity, brainstem, promotion and reversal by modulating $\mathrm{PCO}_{2}$, in newborn primate, 6

Blood-brain barrier, LPS-induced opening, in newborn pigs, 182

Blood flow, cerebral

effects of low-dose dopamine infusion on, in preterm neonates, 742

post-ischemic $\mathrm{CO}_{2}$ response, PEG-SOD and, 530

regional, after fetal asphyxia, flunarizine and, 379

velocity

developmental and physiologic changes in, 385

influence of $\mathrm{MABP}, \mathrm{PCO}_{2}$, and $\mathrm{PO}_{2}$ on, in preterm infants, 173

Blood pressure

mean arterial, influence on cerebral blood flow velocity in preterm infants, 173

systemic, flunarizine and, after fetal asphyxia, 379

Body fat, in infants, 448

Bowel injury, PAF-induced

ischemic necrosis, 237

nitric oxide in, 222

Bradycardia, in preterm infants, 144

Brain: see also Cerebral newborn, indomethacin and, 178

sulfonylurea receptors in, effect of hypoxia on, in rat, 634

superoxide anion generation, in asphyxia, 366

Brain cells

effect of caffeine on, in newborn rat, 716

lactate metabolism in, 709

Brain injury

HIV peptide potentiation of, 192

hypoxic-ischemic

hypothermia and, 525

monosialoganglioside GM1 and, 18

oxygen radical scavengers with magnesium and, 23

Brainstem auditory evoked potentials, reversible, in jaundiced Gunn rats, 629

Brainstem bilirubin toxicity, promotion and reversal by modulating $\mathrm{PCO}_{2}$, in newborn primate, 6

Breastmilk, garlic-flavored, repeated exposure to, effects on nursling's behavior, 805

Breathing: see Fetal breathing

Bronchial reactivity, 478

Bronchopulmonary dysplasia, pulmonary macrophages in, 341

$\gamma$-Butyrobetaine, plasma concentrations and urinary output in drug-induced carnitine depletion, 460

Caffeine, effect on newborn rat brain cultures, 716

Calcium kinetics, pubertal changes, 455

Caloric supplementation, effect on milk factors in undernourished Guatemalan mothers, 217

Carbachol challenge test, interruption technique, 478

Carbon dioxide

partial pressure

influence on cerebral blood flow velocity in preterm infants, 173

modulation of, promotion and reversal of brainstem bilirubin toxicity in newborn primate by, 6

post-ischemic CBF response, PEG-SOD and, 530

ventilatory response to, maturation of, in newborn piglets, 485

Cardiovascular function

effects of low-dose dopamine infusion on, in preterm neonates, 742

response to acute hypoxemia, in fetal lambs, 51

Carnitine

deficiency

secondary, 89

valproic acid-associated, in vitro model of, 281

drug-induced depletion, 460

renal handling in secondary carnitine deficiency disorders, 89

repletion in cystinosis, 115

uptake, effects of valproic acid on, 281

Catalase-deficient peroxisomal particles, with partial deficiency of plasmalogen synthesis, in pseudo infantile Refsum's disease, 270

Catecholamine, plasma levels, effects of low- 


\section{SUBJECT INDEX TO VOLUME 34}

dose dopamine infusion on, in preterm neonates, 742

Celiac disease, fibroblast-derived autoantigens, 420

Cerebral blood flow

effects of low-dose dopamine infusion on, in preterm neonates, 742

post-ischemic $\mathrm{CO}_{2}$ response, PEG-SOD and, 530

regional, after fetal asphyxia, flunarizine and, 379

velocity

developmental and physiologic changes in, 385

influence of $\mathrm{MABP}, \mathrm{PCO}_{2}$, and $\mathrm{PO}_{2}$ on, in preterm infants, 173

Cerebral circulation, effects of $\mathrm{N}$ - $\omega$-nitro-Larginine methylester on, 354

Cerebral glycolytic rates, agonal, during hypercarbia, 370

Cerebral oxygenation, neonatal, determination by near-infrared spectroscopy, effect of fetal $\mathrm{Hb}$ on, 15

Cerebral vascular bed, influence of indomethacin on autoregulatory ability of, in newborn lamb, 178

Cerebrospinal fluid, levels of pterins, HVA, and HIAA, 10

Chemoreceptor responses, in fetus, 51

Cholinephosphate cytidylyltransferase, mass, in developing rat lung, 502

Ciliary dyskinesia, primary, membrane fluidity of polymorphonuclear leukocytes in, 725

Circulation, cerebral, effects of N- $\omega$-nitro-Larginine methylester on, 354

Clinical competencies, self-perceived, in primary care residency graduates, 555

Cocaine, prenatal, effect on striatal dopamine, 389

Cold, response to, in fetal sheep, 47

Colon, infant, influence of acetate on sodium transport in, 666

Colonic acetate, in circulating acetate pool of infant pig, 318

Colostrum, anti-inflammatory activity in, 208

Complement activation, in newborn infants, 199

Complement anaphylatoxin C5a, pulmonary fluid levels, in chronic lung disease. 586

Congenital adrenal hyperplasia, salt-wasting, $3 \beta$-hydroxysteroid dehydrogenase gene mutation in, 698

Congenital heart disease, respiratory mechanics and, 329

Core temperature, transcutaneous, 512

Cystic fibrosis

anti-HSP60 antibodies in, 424

elevated IL-8 with, 159

lipids in, TNF- $\alpha$ and, 162

Cystinosis, nephropathic, muscle carnitine repletion in, 115

Cytidine 5'-triphosphate, immunoreactive, expression of, 502

Cytidylyltransferase, mass, in rat lung, 502

Cytokines

and neonatal polymorphonuclear leukocyte, 249

production in milk cells, 213

Deferoxamine, effect on alveoli formation in rat lung, 334

5-Deiodinase, placental, in selenium-deficient rats, 288

Development: see also Maturation

cerebral blood flow velocity changes, 385 fetal lung, effect of fetal breathing movements on, 148

lung, tissue inhibitor of metalloproteinase1 gene expression in, 729

renal, and $\alpha_{1}$ B-adrenoceptor, 124

Dexamethasone

effects on alveoli formation in rat lung, 334

effects on pulmonary fluid C5a levels in chronic lung disease, 586

Diabetes mellitus

anti-HSP60 antibodies in, 424

insulin-dependent, antibodies to glutamic acid decarboxylase in, 785

insulin-dependent mothers

newborn, postnatal glucose kinetics in, 443

newborn, rates of urea synthesis in, 801 in pregnancy, 791

23,25-Dihydroxyvitamin $D_{3}$, placental synthesis of, 98

24,25-Dihydroxyvitamin $D_{3}$, placental synthesis of, 98

Dopamine

in developing tubule, 308

effect on neonatal growth hormone and prolactin, 642

low-dose infusion, effects on preterm neonates, 742

metabolites, cerebrospinal fluid levels, 10

striatal, effect of prenatal cocaine on, 389

Doppler velocimetry, study of cord compression effects on fetal hemodynamic changes induced by maternal $\mathrm{O}_{2}$ administration in sheep, 796

Drug-induced carnitine depletion, 460

EGF: see Epidermal growth factor

Elastic unloading, ventilation with, 600

Embryo, chick, hemodynamics of, 44

Endothelial cells, reactive oxygen metabolites in, nucleotide depletion due to, 572

Endothelin, effects on renal function, in newborn rabbits, 120

Endothelium-derived nitric oxide

effects on renal hemodynamics and function in sheep fetus, 755

effects on renal hemodynamics in developing piglet, 750

End-systolic pressure-volume relationship, in acidemic fetal sheep, 38

Enterocolitis, necrotizing

prevention of, role of PAF-acetylhydrolase in, 237

research agenda for, 701

Eosinophils, fetal, L-selectin expression of, 253

Epidermal growth factor, effect on fetal lung antioxidant enzymes during hyperoxia, 577

Erythrocytes, neonatal, mechanical and geometrical properties of, 688

Erythropoietin, treatment of anemia of prematurity with, 675

Escherichia coli

0111 B4 lipopolysaccharide, blood-brain barrier opening induced by, in new. born pigs, 182

meningitis, neurotransmitters in, 187

Ether vapor, pituitary-adrenal response to, in weanling animal, 646

Fähraeus effect, of neonatal red blood cell suspensions, 538

Fähraeus-Lindqvist effect, of neonatal red blood cell suspensions, 538

Familial defective apo B, molecular genetics of, 403 renal hemodynamics, 750
Familial hypercholesterolemia, molecular genetics of 403

Fatal infection, anti-TNF antibodies and nitric oxide suppression in rat model, 345

Fatty acid oxidation

contribution to ATP production in neonatal hearts, 735

in pseudo infantile Refsum's disease, 270

Fetal breathing, inhibition of, pilot study, 834

Fetal breathing movements, effect on fetal lung development, 148

Fetal heart rate, fluctuations, spectral analysis of, 134

Fetal medicine, APS Presidential Address, 393

Fetus

asphyxia, flunarizine after, neuroprotective properties of, 379

autonomic state, spectral analysis of, 134

chemoreceptor responses in, 51

eosinophils, L-selectin expression of, 253

glucose, regulation of fetal plasma IGF-I by, 62

granulocytes, L-selectin expression of, 253

hemodynamic changes induced by maternal $\mathrm{O}_{2}$ administration, effects of cord compression on, Doppler study, 796

hemoglobin, and NIRS neonatal monitoring, 15

hypoxemia, acute, cardiovascular response to, in lambs, 51

insulin effect in, ontogeny of, 654

lung

antioxidant enzymes, effect of EGF on, during hyperoxia, 577

development of, effect of fetal breathing movements on, 148

macrosomia, in offspring of rats subjected to protein-energy malnutrition, 791

neutrophils, L-selectin expression of, 253

renal function and hemodynamics, effects of endothelium-derived nitric oxide on, in sheep, 755

selenium-deficient, thyroid hormone economy in, in rats, 288

Fibroblast-derived celiac disease autoantigens, purification of, 420

Flunarizine, neuroprotective properties of, after fetal asphyxia, 379

Follicle-stimulating hormone, bioactive, in girls during puberty, 829

Formyl-methionyl-leucyl-phenylalanine binding studies, of priming of newborn polymorphonuclear neutrophilic granulocytes, 243

Galactose-1-phosphate uridyltransferase, rat, modulation of, 416

Garlic-flavored milk, repeated exposure to, effects on nursling's behavior, 805

Gas exchange, effects of surfactant treatment on, 495

Genetics

of hyperlipidemia, 403

of Pearson's syndrome, 105

Glial cells, effect of caffeine on, in newborn rat, 716

Glucocorticoids, inhibitory effect on adrenocorticotropin secretion, 646

Glucose

fetal, regulation of fetal plasma IGF-I by, 62

oxidation, contribution to ATP production in neonatal hearts, 735

postnatal kinetics in IDM, 443

$\beta$-Glucuronidase, recombinant, enzyme re- 
placement with, in mucopolysaccharidosis VII, 837

Glutamic acid decarboxylase, antibodies to, in insulin-dependent diabetes mellitus, 785

Glycogen storage disease type $1 \mathrm{~b}$, monocytederived macrophage respiratory burst defect, interferon- $\gamma$ correction of, 265

Glycolysis

cerebral agonal rates, during hypercarbia, 370

contribution to ATP production in neonatal hearts, 735

Gonadotropin, concentration profiles in boys, 229

Granulocyte colony-stimulating factor gene expression, in activated cord versus adult mononuclear cells, 560

Granulocyte-macrophage colony-stimulating factor gene expression, in activated cord versus adult mononuclear cells, 560

Granulocytes, fetal, L-selectin expression of, 253

Growth and development: see Development

Growth hormone

neonatal, dopamine effect on, 642

placental, and intrauterine growth retardation, 439

resistance, in HIV-1 infection, 66

Heart disease, congenital, respiratory mechanics and, 329

Heart rate: see also Fetal heart rate

dynamics during infancy, 618

Hearts

ischemic, age-dependency of L-propionylcarnitine on, 465

neonatal

adenosine triphosphate production in, source of, 735

effects of pituitary adenylate cyclase activating polypeptide in, in pig, 323

60-kd Heat shock protein, human, antibodies to, in juvenile chronic arthritis, 424

Hematopoiesis, neonatal, IL-1I and, 56

Hemodynamics

chick embryo, 44

fetal, effects of cord compression on changes induced by maternal $\mathrm{O}_{2}$ administration, Doppler study in sheep, 796

renal, effects of endothelium-derived nitric oxide on

in developing piglet, 750

in sheep fetus, 755

Hemoglobin, fetal, and NIRS neonatal monitoring, 15

Hepatitis B virus, hormonal regulation of, 300

HIV: see Human immunodeficiency virus

Homovanillic acid, cerebrospinal fluid levels, 10

Howland Award

acceptance address, 552

presentation to Barness, 551

Human immunodeficiency virus-derived peptide, potentiation of brain injury by, 192

Human immunodeficiency virus infection congenital, immune complexes in, 781 decreased oxidative burst in, 544

Human immunodeficiency virus- 1 infection, hormone resistance in, 66

Human leukocyte antigen-DR, increased expression on pulmonary macrophages in bronchopulmonary dysplasia, 341
Hyaline membrane disease, effects of surfactant treatment on gas exchange in newborn premature infants with, 495

Hyaluronan secretion, effect of caffeine on, in newborn rat, 716

Hydrogen peroxide, decreased production in HIV-infection, 544

D-2-Hydroxyglutaric acid, stable-isotope dilution analysis of, 277

L-2-Hydroxyglutaric acid, stable-isotope dilution analysis of, 277

D-2-Hydroxyglutaric acidemia, detection and prenatal diagnosis of, 277

L-2-Hydroxyglutaric acidemia, detection and prenatal diagnosis of, 277

5-Hydroxyindoleacetic acid, cerebrospinal fluid levels, 10

$\beta$-Hydroxysteroid dehydrogenase gene mutation, in salt-wasting $\mathrm{CAH}, 698$

Hypercapnic cerebral blood flow, post-ischemic, PEG-SOD and, 530

Hypercarbia

agonal cerebral glycolytic rates during, 370

and bilirubin toxicity, 6

Hypercholesterolemia, familial, molecular genetics of, 403

Hyperinsulinism, in offspring of rats subjected to protein-energy malnutrition, 791

Hyperlipidemia, genetics of, 403

Hyperoxia

effect of EGF on fetal lung antioxidant enzymes during, 577

effect on alveoli formation in rat lung, 334

oxidant stress responses in premature infants during, 360

Hypertension, pulmonary, lung bactericidal activity and, 32

Hyperthermia, apnea and REM sleep excess in piglet during recovery from, 518

Hypobetalipoproteinemia, molecular genetics of, 403

Hypothermia, and hypoxic-ischemic brain damage, 525

Hypothyroidism

congenital, somesthetic pathway and treatment in first year, 73

jejunal function in, 79

Hypoxanthine, concentrations, in intermittent versus continuous hypoxemia, 767

Hypoxemia

acute

effects on laryngeal reflex apnea in lambs, 813

fetal cardiovascular response to, in lambs, 51

chronic, myocardial metabolism in, in lambs, 471

intermittent versus continuous, hypoxanthine, xanthine, and uric acid concentrations in, 767

prolonged

effects on laryngeal reflex apnea in lambs, 813

effects on ventilatory response to changes in $\mathrm{PaO}_{2}$ in lambs, 821

relationship between bradycardia and apnea and, in preterm infants, 144

Hypoxia, chronic, effect on sulfonylurea receptors in rat brain, 634

Hypoxic acidemia, effects on left ventricular end-systolic elastance in fetal sheep 38

Hypoxic-ischemic brain damage

hypothermia and, 525

monosialoganglioside GM1 and, 18 oxygen radical scavengers with magnesium and, 23

Hypoxic vasoconstriction, effect of $\mathrm{N} \omega$-nitro$\mathrm{L}$-arginine methylester on, in neonatal pig lung, 349

Immune complexes, in juvenile rheumatoid arthritis and AIDS, 781

Immunoglobulin G-containing high-molecular-weight complexes, in juvenile rheumatoid arthritis and AIDS, 781

Immunoglobulins, production of, enhancement by human cord blood lymphocytes, 565

Indomethaci

and newborn brain, 178

therapeutic dose, effect on brain superoxide anion generation in asphyxia, 366

Infants: see also Neonates

appropriate for gestational age, placental transport in, 661

body fat in, 448

colon, influence of acetate on sodium transport in, 666

colonic acetate in circulating acetate pool, in pigs, 318

of diabetic mothers postnatal glucose kinetics in, 443

rates of urea synthesis in, 801

heart rate dynamics in, 618

nursling behavior, effects of repeated exposure to garlic-flavored milk on, 805

premature

bradycardia, apnea, and hypoxemia in 144

of less than 33 weeks' gestation, cerebral blood flow velocity, influence of $\mathrm{MABP}, \mathrm{PCO}_{2}$, and $\mathrm{PO}_{2}$ on, 173

of less than 35 weeks' gestational age, sleep state organization in, 624

oxidant stress in, 360

with hyaline membrane disease, effects of surfactant treatment on gas exchange in, 360

respiratory mechanics, before and after left-to-right shunts, 329

small for gestational age

placental transport in, 661

rates of urea synthesis in, 801

urinary insulin-like growth factor-II output in, 435

very low birth weight, selenium status of 293

Infection

early onset, complement activation in newborn infants with, 199

fatal, anti-TNF antibodies and nitric oxide IIV suppression in rat model, 345

congenital, immune complexes in, 781

decreased oxidative burst in, 544

HIV-1, hormone resistance in, 66

respiratory syncytial virus, antibody treatment of, 167

Inflammation, rat subcutaneous air pouch model, anti-inflammatory activity in human colostrum in, 208

Insulin-dependent diabetes mellitus

antibodies to glutamic acid decarboxylase in, 785

newborns of mothers with postnatal glucose kinetics in, 443 rates of urea synthesis in, 801

Insulin effect, fetal, ontogeny of, 654

Insulin-like growth factor-I

plasma, fetal glucose regulation of, 62

resistance in HIV-1 infection, 66 
Insulin-like growth factor-II, urinary output, in infants and children, 435

Insulin-like growth factor II gene expression, in fetal lungs, fetal breathing movements and, 148

Insulin resistance, in HIV-1 infection, 66

Interferon- $\gamma$, and glycogen storage disease type lb monocyte-derived macrophage respiratory burst, 265

Interleukin-8

elevated concentrations, with cystic fibrosis, 159

induction of expression in neuroblastoma cells by retinoic acid, effect of leukocyte chemotaxis on, 720

Interleukin-1, elevated expression in neonatal neutrophils, 249

Interleukin-3, gene expression, 560

Interleukin-11, and neonatal hematopoiesis, 56

Intrapulmonary arteries, adrenoreceptor maturation in, 591

Intratracheal pulmonary ventilation, technique, 606

Intrauterine growth retardation, placental growth hormone and, 439

Iron deficiency, in adolescents, 680

Iron status, serum transferrin receptor assessment of, in prepubertal boys, 297

Ischemia, $\mathrm{CBF} \mathrm{CO}_{2}$ response, $\mathrm{PEG}-\mathrm{SOD}$ and, 530

Islet hyperplasia, in offspring of rats subjected to protein-energy malnutrition, 791

Jaundice, reversible BAEP in Gunn rats with, 629

Jejunal function, in hypothyroid rats, 79

Juvenile chronic arthritis, anti-HSP60 antibodies in, 424

Juvenile rheumatoid arthritis

immune complexes in, 78

$\alpha_{2}$-MG complexes in, 204

Kidney: see Renal

KP-1 cells, 258

Lactate metabolism, regulation by albumin, 709

Lactate oxidation, contribution to ATP production in neonatal hearts, 735

Laryngeal reflex apnea, effects of hypoxemia on, in lambs, 813

Left-to-right shunts, respiratory mechanics before and after, 329

Left ventricular end-systolic elastance, effects of hypoxic acidemia on, in fetal sheep, 38

Left ventricular oxygen and substrate uptake, in chronically hypoxemic lambs, 471

Leukemia, KP-1 monocytic cell lines, 258

Leukocytes

chemotaxis, effect on retinoic acid-induced neuroblastoma cell differentiation, 720

polymorphonuclear

membrane fluidity in primary ciliary dyskinesia, 725

neonatal, cytokines and, 249

Lipid disorders, molecular genetics of, 403

Lipids, in cystic fibrosis, TNF- $\alpha$ and, 162

Lipopolysaccharide

blood-brain barrier opening induced by, in newborn pigs, 182

priming of newborn polymorphonuclear neutrophilic granulocytes with, 243

Lipoprotein(a)

in children from infarction kindreds, 670

serum levels, in chronic renal disease, 772
Lipoprotein lipase deficiency, molecular genetics of, 403

Liposomal zinc protoporphyrin, targeted to spleen, using reticuloendothelial blockade with blank liposomes, 1

Long-chain acyl-CoA dehydrogenase deficiency, 111

Lung: see also Pulmonary

alveoli formation in, in rats, 334

bactericidal activity, and pulmonary hypertension, 32

cytidylyltransferase mass in, in developing rat, 502

development of, tissue inhibitor of metaletal loproteinase-1 gene expression in, 729

antioxidant enzymes, effect of EGF on, during hyperoxia, 577

development of, effect of fetal breathing movements on, 148

neonatal, effect of $\mathrm{N} \omega$-nitro-L-arginine methylester on hypoxic vasoconstriction in, in pig, 349

perinatal, antioxidant enzyme gene expression in, in rat, 27

Lung disease, chronic, pulmonary fluid C5a levels, 586

Luteinizing hormone

bioactive, in girls during puberty, 829

and testosterone, temporal relationship between, 229

Lymphocytes, human cord blood, enhancement of immunoglobulin production by, 565

Lysine, plasma concentrations and urinary output in drug-induced carnitine de. pletion, 460

$\alpha_{2}$-Macroglobulin, intraarticular complexes, in juvenile rheumatoid arthritis, 204

Macrophage colony-stimulating factor gene expression, in activated cord versus adult mononuclear cells, 560

Macrophages

glycogen storage disease type lb monocytederived, respiratory burst defect in, interferon- $\gamma$ correction of, 265

pulmonary, in bronchopulmonary dysplasia, 341

scavenger receptor, human, new monocyte cell line with, 258

Macrosomia, fetal, in offspring of rats subjected to protein-energy malnutrition, 791

Magnesium, oxygen radical scavengers in combination with, and hypoxic-ischemic brain damage, 23

Malnutrition, maternal, pregnancy after, 791

Maturation: see also Development

of adrenoreceptor, intrapulmonary, 591

of ventilatory response to carbon dioxide in newborn piglets, 485

Mean arterial blood pressure, influence on cerebral blood flow velocity in preterm infants, 173

Mechanical ventilation, assisted, with elastic unloading, 600

Medium-chain acyl-coenzyme A dehydrogenase, mutant, expression and characterization of, 694

Meningitis

E. coli, neurotransmitters in, 187

neonatal, LPS-induced blood-brain barrier opening during, 182

Metabolic system, responses to cold, in feta sheep, 47

Metalloproteinase-1, tissue inhibitor of, gene expression in lung development, 729
$N$-Methyl-D-aspartate-mediated brain injury, HIV peptide potentiation of, 192

Mevalonate kinase deficiency, 129

Microbicidal activity, lung, and pulmonary hypertension, 32

Milk cells, cytokine production in, 213

Milk factors, undernutrition and, 217

Mixed connective tissue disease, anti-HSP60 antibodies in, 424

Molecular analyses

genetics of pediatric lipid disorders, 403

of Pearson's syndrome, 105

Monocyte-derived macrophage, glycogen storage disease type $1 \mathrm{~b}$, respiratory burst defect in, interferon- $\gamma$ correction of, 265

Monocytes, decreased superoxide anion and hydrogen peroxide production in HIV-infection, 544

Monocytic leukemia cell lines, KP-1 (with scavenger receptor), 258

Mononuclear cells, activated cord versus adult, transcriptional rates of $\mathrm{GM}$ CSF, G-CSF, IL-3, and M-CSF genes in, 560

Monosialoganglioside GMI, and hypoxic-ischemic brain damage, 18

Mucopolysaccharidosis VII, enzyme replacement in, 837

Muscle carnitine, repletion in cystinosis, 115

Myocardial infarction, premature, parental, lipoprotein(a) in children from kindreds with, 670

Myocardial ischemia, control by L-propionylcarnitine, 465

Myocardial metabolism, in chronically hypoxemic lambs, 471

Near-infrared spectroscopy

neonatal monitoring, fetal $\mathrm{Hb}$ and, 15

quantification of cerebral effects of $\mathrm{N}-\omega$ nitro-L-arginine methylester in newborn piglets by, 354

Necrotizing enterocolitis

prevention of, role of PAF-acetylhydrolase in, 237

research agenda for, 701

Neonates: see also Premature infants

asphyxic, resuscitation with $21 \%$ or $100 \%$ $\mathrm{O}_{2}, 809$

brain, indomethacin and, 178

brain cells, effect of caffeine on, in rat, 716 cerebral circulation, effects of $\mathrm{N}$ - $\omega$-nitro-L arginine methylester on, in piglets, 354

cerebral oxygenation, determination by near-infrared spectroscopy, effect of fetal $\mathrm{Hb}$ on, 15

complement activation in, 199

dopamine effect on growth hormone and prolactin in, 642

heart

adenosine triphosphate production in source of, 735

effects of pituitary adenylate cyclase activating polypeptide in, in pigs, 323

hematopoiesis, IL-11 and, 56

of insulin-dependent diabetic mothers, postnatal glucose kinetics in, 443

lung, effect of $\mathrm{N} \omega$-nitro-L-arginine methylester on hypoxic vasoconstriction in, in pigs, 349

meningitis, experimental, LPS-induced blood-brain barrier opening during, 182

mucopolysaccharidosis VII, enzyme replacement in, in mice, 837 
neutrophils, elevated IL-1 expression in, 249

polymorphonuclear leukocyte, cytokines and, 249

polymorphonuclear neutrophilic granulocyte, priming of, 243

pulmonary capillary recruitment in, in lambs, 596

purine and pyrimidine excretion in, 762

red blood cell mechanics in, 688

red blood cell suspensions, Fåhraeus and Fähraeus-Lindqvist effects of, 538

renal function, effects of endothelin on, in rabbits, 120

urea synthesis rates in, 801

vagal response, time-dependent changes in, 139

ventilatory response to carbon dioxide, maturation of, in piglets, 485

Nephropathic cystinosis, muscle carnitine repletion in, 115

Nephrotic syndrome

oligosaccharides in, 429

peritonitis associated with, role of $I$ and $B$ in, 84

Neuroblastoma cells, retinoic acid-induced differentiation, effect of leukocyte chemotaxis on, 720

Neurotransmitters, in E. coli meningitis, 187 Neutrophils

decreased superoxide anion and hydrogen peroxide production in HIV-infection, 544

fetal, L-selectin expression of, 253

neonatal, elevated IL-1 expression in, 249

Newborn: see Neonates

Nitric oxide

endothelium-derived

effects on renal hemodynamics and function in sheep fetus, 755

effects on renal hemodynamics in developing piglet, 750

in PAF-induced bowel injury, 222

suppression of, anti-TNF antibodies and, 345

$\mathrm{N}$ - $\omega$-nitro-L-arginine methylester

cerebral effects, 354

effect on pulmonary circulation, 349

Nucleotides

depletion due to reactive oxygen metabolites in endothelial cells, 572

immunomodulating actions of, 565

Nurslings, behavior of, effects of repeated exposure to garlic-flavored milk on, 805

Nutrition, Howland Award acceptance address, 552

Oligohydramnios, respiratory function after, 611

Oligosaccharides, in nephrotic syndrome, 429

Ornithine transcarbamylase, congenital deficiency, peripheral-type benzodiazepine sites in, 777

Oxidant stress, in premature infants, 360

Oxidative burst, decreased, in HIV-infection, 544

Oxygen

arterial partial pressure, changes in, effect of prolonged hypoxemia on ventilatory response to, in lambs, 821

maternal administration of, fetal hemodynamic changes induced by, effects of cord compression on, Doppler study, 796

metabolites, reactive, in endothelial cells, nucleotide depletion due to, 572
$21 \%$ or $100 \%$, resuscitation of asphyxic newborn infants with, 809

partial pressure, influence on cerebral blood flow velocity in preterm infants, 173

Oxygenation, cerebral, neonatal, determination by near-infrared spectroscopy, effect of fetal $\mathrm{Hb}$ on, 15

Oxygen radical scavengers, in combination with magnesium, and hypoxic-ischemic brain damage, 23

Pearson's syndrome, molecular and genetic analyses of, 105

Peptide, HIV, potentiation of brain injury by, 192

Peritonitis, proteins I and B in, 84

Peroxisomal particles, catalase-deficient with partial deficiency of plasmalogen synthesis, in pseudo infantile Refsum's disease, 270

Pituitary adenylate cyclase activating polypeptide, effects in neonatal pig hearts, 323

Pituitary-adrenal axis, response to ether vapor, in weanling animal, 646

Placenta

5-deiodinase activity, in selenium-deficient rat, 288

growth hormone, and intrauterine growth retardation, 439

$24,25(\mathrm{OH})_{2} \mathrm{D}_{3}$ and $23,25(\mathrm{OH})_{2} \mathrm{D}_{3}$ synthesis, 98

Placental transport, in appropriate and small for gestational age babies, 661

Plasmalogen synthesis, catalase-deficient peroxisomal particles with partial deficiency of, in pseudo infantile Refsum's disease, 270

Platelet-activating factor-acetylhydrolase, role in prevention of necrotizing enterocolitis, 237

Platelet-activating factor-induced bowel injury

ischemic necrosis, 237

nitric oxide in, 222

Polyethylene glycol-conjugated superoxide dismutase, and post-ischemic CBF $\mathrm{CO}_{2}$ response, 530

Polymorphonuclear leukocytes

membrane fluidity of, in primary ciliary dyskinesia, 725

neonatal, cytokines and, 249

Polymorphonuclear neutrophilic granulocyte, newborn, priming of, 243

Pregnancy, after maternal malnutrition, 791

Premature infants

bradycardia, apnea, and hypoxemia in, 144

effects of low-dose dopamine infusion on, 742

of less than 33 weeks' gestation, cerebral blood flow velocity, influence of $\mathrm{MABP}, \mathrm{PCO}_{2}$, and $\mathrm{PO}_{2}$ on, 173

of less than 35 weeks' gestational age, sleep state organization in, 624

oxidant stress in, 360

with hyaline membrane disease, effects of surfactant treatment on gas exchange in, 360

Prematurity, anemia of, erythropoietin treatment in, 675

Primary care residency graduates, clinical competencies in, 555

Prolactin, neonatal, dopamine effect on, 642

L-Propionylcarnitine, age-dependency of, on ischemic hearts, 465

Protein-energy malnutrition, maternal, pregnancy after, 791
Proteins I and B, role in peritonitis, 84

Proteolytic activity, in juvenile rheumatoid arthritis, 204

Pseudo infantile Refsum's disease, 270

Pterins, cerebrospinal fluid levels, 10

Puberty

bioactive luteinizing and follicle-stimulating hormone in girls during, 829

calcium kinetics changes during, 455

Pulmonary arteries, adrenoreceptor maturation in, 591

Pulmonary capillary recruitment, in neonatal lambs, 596

Pulmonary circulation, effect of N $\omega$-nitro-Larginine methylester on, 349

Pulmonary fluid, C5a levels, in chronic lung disease, 586

Pulmonary hypertension, lung bactericidal activity and, 32

Pulmonary macrophages, in bronchopulmonary dysplasia, 341

Pulmonary ventilation, intratracheal, 606

Purine metabolites, neonatal excretion, 762

Pyrimidine metabolites, neonatal excretion, 762

Rapid eye movement sleep, excess, during recovery from hyperthermia, 518

Red blood cells

neonatal, mechanical and geometrical properties of, 688

suspensions, neonatal, Fähraeus and Fähraeus-Lindqvist effects of, 538

Reflex apnea, laryngeal, effects of hypoxemia on, in lambs, 813

Refsum's disease, pseudo infantile, 270

Regional blood flow, responses to cold, in fetal sheep, 47

Renal cortical and medullary tubule cells, effect of dopamine on adenylate cyclase and $\mathrm{Na}^{+}, \mathrm{K}^{+}-\mathrm{ATPase}$ in, in developing rat, 308

Renal disease, chronic, lipoprotein(a) serum levels in, 772

Renal function

developmental regulation of $\alpha_{1}$ B-adrenoceptor, 124

effects of endothelin on, in newborn rabbits, 120

effects of endothelium-derived nitric oxide on, in sheep fetus, 755

effects of low-dose dopamine infusion on, in preterm neonates, 742

handling of carnitine in secondary carnitine deficiency disorders, 89

Renal hemodynamics, effects of endothelium-derived nitric oxide on

in developing piglet, 750

in sheep fetus, 755

Respiration, during recovery from hyperthermia, 518

Respiratory failure, distribution of exogenous surfactant with, 154

Respiratory function, after oligohydramnios, 611

Respiratory mechanics, before and after leftto-right shunts, 329

Respiratory syncytial virus infection, antibody treatment of, 167

Respiratory syncytial virus-specific immune serum, treatment of respiratory syncytial virus infection with, 167

Resuscitation, with $21 \%$ or $100 \% \mathrm{O}_{2}, 809$

Reticuloendothelial blockade, with blank liposomes, zinc protoporphyrin liposomes targeted to spleen using, 1

Retinoic acid-induced neuroblastoma cell 
differentiation, effect of leukocyte chemotaxis on, 720

Rheumatoid arthritis. see Juvenile rheumatoid arthritis

Scavenger receptor, human macrophage, new monocyte cell line with, 258

L-Selectin, expression of fetal granulocytes, 253

Selenium deficiency, effects on placental 5deiodinase activity and fetal thyroid hormone economy in rat, 288

Selenium status, of very low birth weight infants, 293

Serotonin metabolites, cerebrospinal fluid levels, 10

Serpin, synthesis by trophoblast, 312

Shunts, left-to-right, respiratory mechanics before and after, 329

Sleep, REM, excess, during recovery from hyperthermia, 518

Sleep states, organization in very premature infants, 624

Sleep-waking states, heart rate dynamics during, 618

Small for gestational age infants

placental transport in, 661

rates of urea synthesis in, 801

Society for Pediatric Research, Presidential Address, 397

Sodium,potassium adenosine triphosphatase, in developing tubule, effect of dopamine on, 308

Sodium transport, influence of acetate on 666

Somesthetic pathway, and treatment for congenital hypothyroidism in first year, 73

Spectroscopy

fetal autonomic state by, 134

near-infrared

neonatal monitoring, fetal $\mathrm{Hb}$ and, 15 quantification of cerebral effects of $\mathrm{N}-\omega$ nitro-L-arginine methylester in newborn piglets by, 354

Spleen, zinc protoporphyrin liposomes targeted to, using reticuloendothelial blockade with blank liposomes, 1

Stable-isotope dilution analysis, of $\mathrm{D}$ - and $\mathrm{L}$ 2-hydroxyglutaric acid, 277
Subcutaneous adipose tissue, estimation of in infants, 448

Sulfonamide, effect on BAEP abnormalities in jaundiced Gunn rats, 629

Sulfonylurea receptors, effect of hypoxia on in rat brain, 634

Superoxide, in asphyxia, 366

Superoxide anion, decreased production in HIV-infection, 544

Surfactant

effects on gas exchange, 495

exogenous

distribution of, 154

instillation of, effects of, 490

fetal lung, effect of EGF on, during hyperoxia, 577

Sympathoadrenal system, responses to cold in fetal sheep, 47

Systemic blood pressure, flunarizine and after fetal asphyxia, 379

Systemic lupus erythematosus, anti-HSP60 antibodies in, 424

Temperature, transcutaneous core, 512

Testosterone

concentration profiles in boys, 229

luteinizing hormone and, temporal relationship between, 229

Thyroid hormone, fetal economy, in selenium-deficient rats, 288

Tissue inhibitor of metalloproteinase- 1 gene, expression in lung development, 729

Total body fat, estimation in infants, 448

Transcutaneous core temperature, 512

Transferrin receptor, serum, in prepubertal boys, 297

$\epsilon-\mathrm{N}$-Trimethyllysine, plasma concentrations and urinary output in drug-induced carnitine depletion, 460

Trophoblast, serpin synthesis by, 312

Tubule cells, developing rat renal cortical and medullary, effect of dopamine on adenylate cyclase and $\mathrm{Na}^{+}, \mathrm{K}^{+}$-ATPase in, 308

Tumor necrosis factor- $\alpha$

and lipids in cystic fibrosis, 162

priming of newborn polymorphonuclear neutrophilic granulocytes with, 243

Tumor necrosis factor, antibodies to, and nitric oxide suppression, 345
Ubiquinone-10, decreased plasma concentrations, with mevalonate kinase deficiency, 129

Umbilical cord blood

lymphocytes, enhancement of immunoglobulin production by, 565

mononuclear cells, activated, transcriptional rates of GM-CSF, G-CSF, IL-3, and M-CSF genes in, 560

Umbilical cord blood-derived polymorphonuclear neutrophilic granulocyte, newborn, priming of, 243

Umbilical cord compression, effects on fetal hemodynamic changes induced by maternal $\mathrm{O}_{2}$ administration, Doppler study, 796

Undernutrition, and milk factors, 217

Urea synthesis rates, in newborn infants, 801

Uric acid

concentrations, in intermittent versus continuous hypoxemia, 767

inhibition of xanthine oxidase in human plasma by, 303

Vagal nerve, neonatal, response, time-dependent changes in, 139

Valproic acid, effects on carnitine uptake, 281

Valproic acid-associated carnitine deficiency, in vitro model of, 28

Vasoconstriction, hypoxic, effect of $\mathbf{N} \omega$-nitro-L-arginine methylester on, in neonatal pig lung, 349

Ventilation

with elastic unloading, 600

intratracheal pulmonary, 606

response to carbon dioxide, maturation of in newborn piglets, 485

response to changes in $\mathrm{PaO}_{2}$, in lambs, 821

Very-long-chain acyl-CoA dehydrogenase deficiency, identification of, 111

Very low birth weight infants, selenium status of, 293

Xanthine, concentrations, in intermittent versus continuous hypoxemia, 767

Xanthine oxidase, uric acid inhibition of, in human plasma, 303

Zinc protoporphyrin liposomes, targeted to spleen, using reticuloendothelial blockade with blank liposomes, 